\title{
Risk prediction of biomarkers for early multiple organ dysfunction in critically ill patients
}

\author{
Shigeto Ishikawa ${ }^{1 *}$, Yuto Teshima', Hiroki Otsubo', Takashi Shimazui², Taka-aki Nakada², Osamu Takasu², \\ Kenichi Matsuda ${ }^{4}$, Junichi Sasaki ${ }^{5}$, Masakazu Nabeta ${ }^{3}$, Takeshi Moriguchi ${ }^{4}$, Takayuki Shibusawa ${ }^{5}$, \\ Toshihiko Mayumi ${ }^{1}$ and Shigeto Oda ${ }^{2}$
}

\begin{abstract}
Background: Shock and organ damage occur in critically ill patients in the emergency department because of biological responses to invasion, and cytokines play an important role in their development. It is important to predict early multiple organ dysfunction (MOD) because it is useful in predicting patient outcomes and selecting treatment strategies. This study examined the accuracy of biomarkers, including interleukin (IL)-6, in predicting early MOD in critically ill patients compared with that of quick sequential organ failure assessment (qSOFA).

Methods: This was a multicenter observational sub-study. Five universities from 2016 to 2018. Data of adult patients with systemic inflammatory response syndrome who presented to the emergency department or were admitted to the intensive care unit were prospectively evaluated. qSOFA score and each biomarker (IL-6, IL-8, IL-10, tumor necrosis factor-a, C-reactive protein, and procalcitonin [PCT]) level were assessed on Days 0,1 , and 2. The primary outcome was set as MOD on Day 2, and the area under the curve (AUC) was analyzed to evaluate qSOFA scores and biomarker levels.

Results: Of 199 patients, 38 were excluded and 161 were included. Patients with MOD on Day 2 had significantly higher qSOFA, SOFA, and Acute Physiology and Chronic Health Evaluation II scores and a trend toward worse prognosis, including mortality. The AUC for qSOFA score (Day 0) that predicted MOD (Day 2) was 0.728 (95\% confidence interval [CI]: 0.651-0.794). IL-6 (Day 1) showed the highest AUC among all biomarkers $(0.790$ [95\% Cl: 0.711-852]). The combination of qSOFA (Day 0) and IL-6 (Day 1) showed improved prediction accuracy (0.842 [95\% Cl: 0.771-0.893]). The combination model using qSOFA (Day 1) and IL-6 (Day 1) also showed a higher AUC (0.868 [95\% Cl: 0.799-0.915]). The combination model of IL-8 and PCT also showed a significant improvement in AUC.
\end{abstract}

Conclusions: The addition of IL-6, IL-8 and PCT to qSOFA scores improved the accuracy of early MOD prediction.

Keywords: Critically ill, Interleukin, Multiple organ dysfunction, Predictive marker, qSOFA

\footnotetext{
* Correspondence: shgtishkw@med.uoeh-u.ac.jp

'Department of Emergency Medicine, University of Occupational and Environmental Health, 1-1 Iseigaoka, Yahata-nishi, Kitakyushu 807-8555, Japan Full list of author information is available at the end of the article
}

(c) The Author(s). 2021 Open Access This article is licensed under a Creative Commons Attribution 4.0 International License, which permits use, sharing, adaptation, distribution and reproduction in any medium or format, as long as you give appropriate credit to the original author(s) and the source, provide a link to the Creative Commons licence, and indicate if changes were made. The images or other third party material in this article are included in the article's Creative Commons licence, unless indicated otherwise in a credit line to the material. If material is not included in the article's Creative Commons licence and your intended use is not permitted by statutory regulation or exceeds the permitted use, you will need to obtain permission directly from the copyright holder. To view a copy of this licence, visit http://creativecommons.org/licenses/by/4.0/ The Creative Commons Public Domain Dedication waiver (http://creativecommons.org/publicdomain/zero/1.0/) applies to the data made available in this article, unless otherwise stated in a credit line to the data. 


\section{Background}

Excessive immune response with the overproduction of inflammatory mediators leads to systemic inflammatory response syndrome (SIRS), which is crucial for the development of multiple organ dysfunction (MOD) [1]. MOD is an independent prognostic factor for intensive care unit (ICU) mortality [2]. Patients with MOD have longer ICU stays and higher mortality rates $[3,4]$. The early detection of MODS may help identify patients at a risk of prolonged illness and death. Therefore, predicting early MOD can improve patient outcomes and quality of care.

Sequential organ failure assessment (SOFA) is a scale used to score organ failure and predict mortality by determining disease severity [5]. However, blood tests, including those of arterial blood gases, are essential to assess SOFA. In contrast, quick SOFA (qSOFA) was designed as a simple tool that can be used in the emergency department (ED) and can be used without performing blood tests [6]. qSOFA is considered a useful index for identifying patients with high mortality and those requiring systemic management in the ICU and is highly convenient in primary care [7]. In addition, the in-hospital mortality predictive effectiveness of qSOFA was statistically higher than that of SOFA in cases of suspected infection outside the ICU [8]. However, qSOFA did not outperform SOFA for patients in the ICU. qSOFA is affected by the severity of infection and quality of the health care system, and factors such as biomarkers that correlate with systemic inflammation are not included in the score. Therefore, the addition of simple blood tests, including those of cytokines, as in the present study, may improve the diagnostic accuracy of qSOFA for sepsis [9-11].

Interleukin (IL)- 6 is a proinflammatory cytokine released by immune cells and reflects the degree of hypercytokinemia involved in systemic inflammatory changes [12]. IL-6 peaks at $6 \mathrm{~h}$ after invasion and is induced earlier than $\mathrm{C}$-reactive protein (CRP) and procalcitonin (PCT). Furthermore, IL-6 enables earlier diagnoses of SIRS, and IL-6 levels reflect the severity and outcome of sepsis [13-15]. For these reasons, we focused on IL-6. We hypothesized that IL- 6 could predict early MOD and that the addition of biomarkers to qSOFA could improve the prediction accuracy. The objective of this study is to investigate the early MOD prediction of biomarkers including IL-6 and the improvement of prediction accuracy by combining qSOFA with biomarkers.

\section{Methods}

\section{Settings}

This study was conducted using data from EDs and ICUs from 5 university hospitals (University of Occupational and Environmental Health, Chiba University, Kurume University, University of Yamanashi and Keio
University) in Japan as a secondary analysis of a study [16]. The mother study aimed to identify the biomarker with the highest predictive value for late-phase MOD in critically ill patients. Various biomarkers were measured at three timepoints (days 0, 1, and 2). They evaluated predictive values for MOD (primary outcome, MOD on day 7 [late-phase]; secondary outcome, MOD on day 3 [early-phase]). This study was approved by the Institutional Review Board of the University of Occupational and Environmental Health (approval number H28-120).

\section{Study population}

This study included patients with SIRS between September 2016 and September 2018. Patients were enrolled consecutively. The inclusion criteria were patient emergency admission (ICU or ED), age $\geq 20$ years at the time of obtaining consent, patients predicted to hospitalized for at least $48 \mathrm{~h}$ by each physician in charge and patients with a diagnosis of SIRS according to the American College of Chest Physicians/Society of Critical Care Medicine criteria on admission [17]. In cases consent could not be obtained from the patient himself/herself due to impaired consciousness or dementia, consent was obtained from a spouse, relative, or other substitute. Patients with trauma were included if they had multiple injuries ( $\geq 2$ injured area) and had a predicted Injury Severity Score $\geq 10$, and patients with burns were included if they had a Burn Index $\geq 15$. Patients who received steroids, immunosuppressive drugs, or preparations that affected serum IL- 6 concentration within 1 week before emergency transport or ICU admission, those with HIV infection, those who were pregnant, or those considered ineligible for enrollment were excluded.

\section{Data collection}

The patient information included demographic characteristics, etiology of admission, comorbidities, presence of hemodynamic instability, and history of treatment in the ED/ICU. Blood samples were collected $6 \mathrm{~h}$ after admission (Day 0) and the following morning (Day 1). Blood samples on Day 2 were collected during the morning period. We measured a suite of biomarkers to see which ones improved prediction. The inflammatory biological markers included CRP, IL-6, IL-8, IL-10, tumor necrosis factor (TNF)- $\alpha$, and PCT. For biomarkers other than IL-6, we selected them that have been widely studied as markers of inflammation [18-22]. Serum CRP levels were measured immediately using commercially available assays at each hospital; IL, TNF$\alpha$, and PCT levels were measured at outside facilities after serum samples were frozen and stored at $-20^{\circ} \mathrm{C}$ (IL-6 and PCT were measured at Roche diagnostics K.K., TNF- $\alpha$, IL-8, IL-10 were measured at SRL,inc). 
The biomarkers were determined using reagent kit for IL-6 and PCT (Elecsys IL-6 and Elecsys BRAHMS PCT), Roche Diagnostics GmbH, Mannheim, Germany; IL-8 and IL-10 (BIOSOURCE IL-8 EASIA kit and BIOSOURCE IL-10 EASIA kit), BioSource Europe S.A., Nivelles, Belgium; TNF- $\alpha$ (Quantikine HS ELISA Human TNF- $\alpha$ Immunoassay), R\&D Systmes, Inc., Minneapolis USA. MOD was defined as two or more organs with a SOFA score $\geq 2$. qSOFA and SOFA scores were recorded on Days 0, 1, and 2. The Acute Physiology and Chronic Health Evaluation (APACHE) II scores were recorded on admission.

\section{Statistical analysis}

The outcome as an early MOD was assessed on Day 2 because reversible derangements induced by the inciting event or incomplete resuscitation may be reflected $24 \mathrm{~h}$ after admission. To evaluate the accuracy of predicting MOD by adding biomarkers to qSOFA, we developed a baseline model to predict MOD (Day 2) using a logistic regression analysis. We calculated the area under the curve (AUC) by drawing an ROC curve for the blood concentration of each biomarker on Days 0 and 1 to determine which biomarker and time points were most predictive of MOD on Day 2. We did a subgroup analysis with groups such as ED/ICU and infection/trauma/burn. The biomarkers and days with the highest AUC were used in the model added to qSOFA to evaluate whether the model improves the accuracy of predicting MOD. ROC curves were drawn, and AUC values were compared between the baseline model and the model with the biomarker added to qSOFA. The calculation of the $95 \% \mathrm{CI}$ of AUC and testing for differences in AUC were performed based on the method of DeLong [23]. We calculated the net reclassification improvement (NRI) to compare each model and presented the values with 95\% confidence intervals (CIs) [24]. The results were compared using Mann-Whitney $\mathrm{U}$ tests and chi-square tests. Multiple testing correction was applied using Sidak's correction. P-values < 0.05 were considered statistically significant. All statistical analyses were performed using $\mathrm{R}$ version 3.5.3 and JMP 13.2.1. This study was analyzed by an independent statistician.

\section{Results}

Of 199 patients enrolled in the study: 5 who withdrew their consent, 4 who were on steroids before admission, 7 who died within $48 \mathrm{~h}$ of admission, 1 who was discharged within $48 \mathrm{~h}$ of admission, and 21 who had deviated from the protocol for biomarker testing and SOFA score calculation due to missing samples or improper timing of the sampling. These 31 patients were excluded
(Fig. 1). Of 161 eligible patients, 99 patients had MOD on Day 0. On Day 1, 9 patients recovered from MOD and 12 patients developed new MOD, for a total of 102 patients with MOD. On Day 2, 7 patients recovered from MOD and 1 patient had new MOD, for a total of 96 patients with MOD. No patients died by Day 2. Patients with MOD had significantly higher qSOFA, SOFA, APACHE II scores, mortality rates, fewer ICU-free days, ventilator-free days, renal replacement therapy (RRT)free days (Table 1).

Each biomarker showed higher values in patients with MOD, except for WBC. CRP and PCT peaked after day 1, while IL-6, IL-8 and IL-10 peaked on Day 0 (Table 2). The ROC analysis of each biomarker on Day 0 and 1 was performed to assess MOD on Day 2. The results showed that IL-6 on Day 1 had the highest AUC (AUC: 0.790; 95\% CI: 0.711-0.852), followed by IL-8 (AUC: 0.789; 95\% CI: 0.712-0.850) (Table 3). On Day 0, PCT showed the highest AUC (AUC: 0.728; 95\% CI: 0.644-0.799). Subgroup analysis was performed in the same way. In the ICU admission group, PCT on Day 0 and IL8 and IL6 on Day 1 showed high AUC (Additional file 1). In the group with infection, PCT showed the highest AUC on Day 0, IL8 on Day 1, followed by IL6 (Additional file 2). In the group without infection, IL6 on Day 1 showed the highest AUC (Additional file 3). In the group without ICU admission, ROC analysis could not be performed because there was only one case with MOD on Day 2.

Next, as a baseline model, the predictive accuracy of MOD on Day 2 using Day 0 qSOFA scores was evaluated by logistic regression analysis (AUC: 0.728; 95\% CI: 0.651-0.794). We developed a model combining qSOFA with IL-6, IL-8, and PCT, which showed high AUC. PCT was the biomarker that showed the highest AUC in the Day 0 combination (AUC: 0.814; 95\% CI: 0.740-0.870; NRI: 0.661). The addition of Day 0 IL-6 significantly improved the NRI but did not significantly improve the AUC (AUC: 0.765; 95\% CI: 0.685-0.830; NRI: 0.562). We analyzed the predictive accuracy of MOD (Day 2) in several models that individually added IL-6, IL-8, PCT (Day 1) to qSOFA (Day 0). In this additional model, IL-6 had the highest AUC compared to the baseline model (AUC: 0.842; 95\% CI: 0.771-0.893; NRI: 0.802) (Table 4/Fig. 2). We also explored the possibility of further improving our accuracy by measuring qSOFA on Day 1. Changing qSOFA in this model from Day 0 to Day 1 and analyzing it in the same manner resulted in a significant improvement in AUC (AUC: 0.868; 95\% CI: 0.799-0.915; NRI: 0.712). IL-8 and PCT also improved AUC predominantly in the same combination model. 


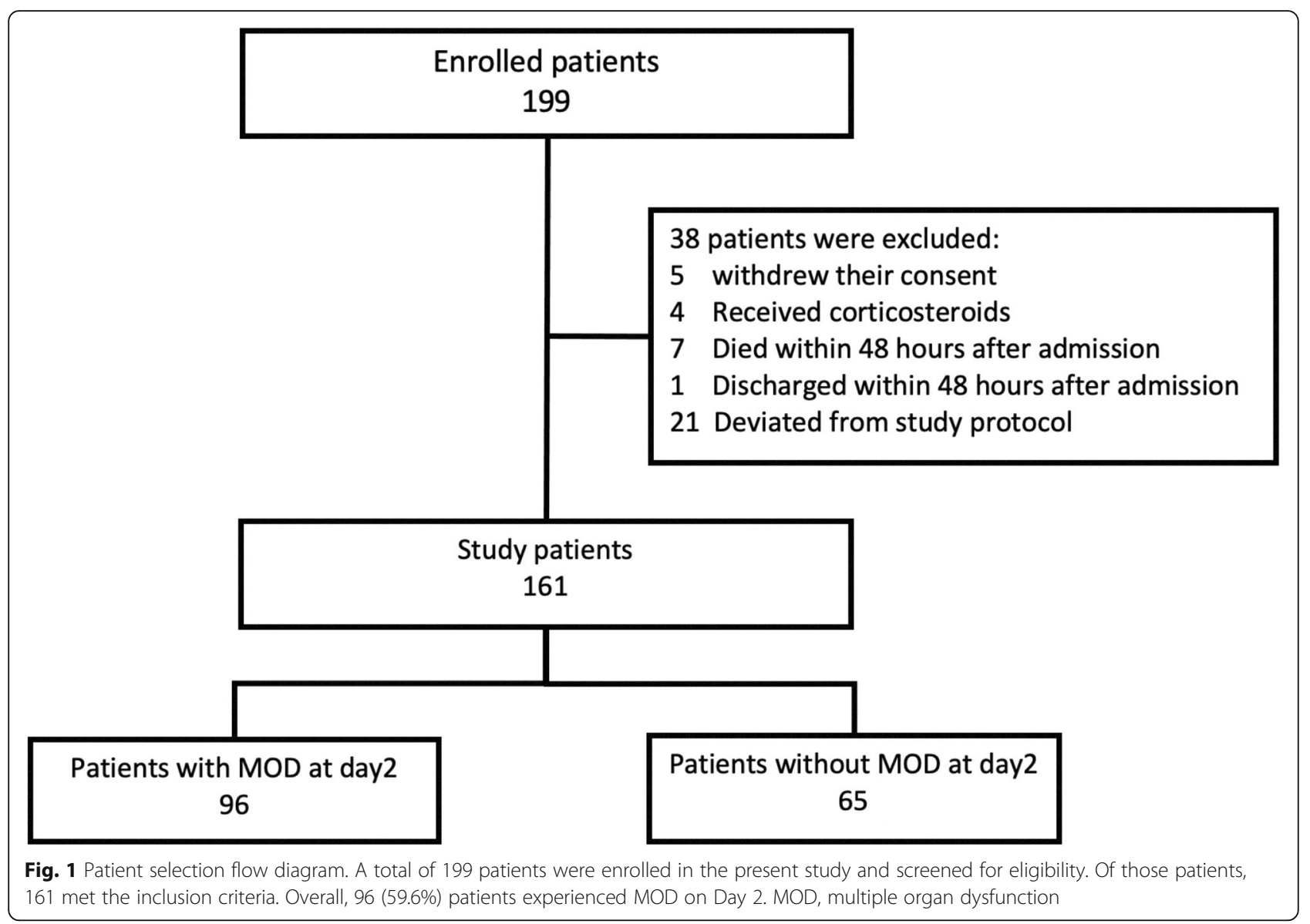

\section{Discussion}

In this study, IL- 6 on Day 1 was the most accurate biomarker for predicting early MOD. Furthermore, the addition of IL-6, IL-8, PCT blood levels to qSOFA was a more accurate predictor of early MOD. Although previous studies implicated that patients with MOD had poor prognosis, including increased mortality and hospitalization days $[2,3]$, this study also confirmed that patients with MOD on Day 2 had poor prognosis (Table 1).

Cytokine storms play an important role in the pathogenesis of MOD, and various cytokines have been assessed for their accuracy in predicting MOD. IL-6 is a cytokine involved in inflammatory responses and is released in response to tissue injuries and inflammatory stimuli, resulting in a physiological response. IL-6 acts locally and systemically and is a proinflammatory mediator and an anti-inflammatory regulator that stimulates anti-inflammatory cytokines, including IL-10 [25]. The mediators of acute inflammation and infection, such as CRP, IL-6, and PCT, have long been involved in the pathophysiology of critically ill patients and are routinely used for diagnostic, prognostic, and treatment monitoring in the ICU [25-27]. Compared with other biomarkers such as CRP and PCT, IL-6 responds more quickly to inflammatory stimuli, peaking at $3-6 \mathrm{~h}$. This suggests that it has an advantage in predicting risk at admission. In this regard, several reports have shown that IL-6 is a useful biomarker for detecting early sepsis; however, CRP and PCT are still often within the reference range owing to their much slower rates [28-30]. On the other hand, some reports suggest that PCT is the predominant marker for the diagnosis of sepsis [31-33]. The AUC in Table 2 revealed that the predictive ability of IL-6 peaked on Day 1, and PCT had a higher AUC than IL-6 on Day 0. The subgroup analysis also showed that the prediction accuracy of PCT was significantly improved in the group with infection. This may indicate that IL- 6 levels have an early increase in the blood but remain more reflective of MOD than other biomarkers after Day 1. In a study examining various cytokine concentrations in patients with severe sepsis, IL-6 and IL- 8 in the first $24 \mathrm{~h}$ predicted organ dysfunction on Day 3 [18]. In light of these findings, the present study shows that IL-6 may predict early MOD with greater accuracy than other biomarkers. 
Table 1 Characteristics and clinical outcomes in patients with or without multiple organ dysfunction on Day2

\begin{tabular}{|c|c|c|c|}
\hline & $\begin{array}{l}\text { MOD } \\
(n=96)\end{array}$ & $\begin{array}{l}\text { without MOD } \\
(n=65)\end{array}$ & $P$ value \\
\hline \multicolumn{4}{|l|}{ Characteristics } \\
\hline Age, years & 70 (59-78) & $72(64-85)$ & 0.145 \\
\hline Male sex, $\mathrm{n}(\%)$ & $59(61.5)$ & $42(64.6)$ & 0.684 \\
\hline \multicolumn{4}{|l|}{ Etiology of SIRS, (\%) } \\
\hline Infection & $63(65.6)$ & $46(70.8)$ & 0.493 \\
\hline Post-surgery & $8(8.3)$ & $4(6.2)$ & 0.605 \\
\hline Trauma & $14(14.6)$ & $8(12.3)$ & 0.680 \\
\hline Burn & $1(1.0)$ & $0(0.0)$ & 0.409 \\
\hline Acute pancreatitis & $6(6.3)$ & $3(4.6)$ & 0.658 \\
\hline Others & $10(10.4)$ & $6(9.2)$ & 0.805 \\
\hline APACHEll score on admission & $29(23-37)$ & $19(13-22)$ & $<0.001^{*}$ \\
\hline qSOFA on admission & & & $<0.001^{*}$ \\
\hline 0 & 1 & 14 & - \\
\hline 1 & 33 & 29 & - \\
\hline 2 & 35 & 21 & - \\
\hline 3 & 26 & 1 & - \\
\hline qSOFA $\geqq 2$ on admission, $\mathrm{n}(\%)$ & $61(64.2)$ & $22(33.8)$ & $<0.001^{*}$ \\
\hline \multicolumn{4}{|l|}{ SOFA score on admission } \\
\hline Total SOFA score & $10(7-13)$ & $3(2-5)$ & $<0.001^{*}$ \\
\hline Respiration, (\%) & $72(75.0)$ & $23(37.1)$ & $<0.001^{*}$ \\
\hline Coagulation, (\%) & $29(30.2)$ & $4(6.2)$ & $<0.001^{*}$ \\
\hline Liver, (\%) & $14(14.7)$ & $4(6.2)$ & 0.092 \\
\hline Cardiovascular, (\%) & $58(60.4)$ & $3(4.6)$ & $<0.001^{*}$ \\
\hline Central Nervous System, (\%) & $67(69.8)$ & $9(13.8)$ & $<0.001^{*}$ \\
\hline Renal, (\%) & $46(47.9)$ & $9(13.8)$ & $<0.001^{*}$ \\
\hline \multicolumn{4}{|l|}{ Outcome } \\
\hline In hospital 28 day mortality & $16(16.7)$ & $2(3.1)$ & $0.007^{*}$ \\
\hline ICU free days & $13(0-20)$ & $26(23-28)$ & $<0.001^{*}$ \\
\hline Ventilator free days & $16(1-24)$ & $28(28-28)$ & $<0.001^{*}$ \\
\hline RRT free days & $25(11-28)$ & $28(28-28)$ & $<0.001^{*}$ \\
\hline
\end{tabular}

MOD Multiple organ dysfunction, SIRS Systemic inflammatory response syndrome, APACHE Acute Physiology and Chronic Health Evaluation, qSOFA Quick Sequential Organ Failure Assessment, ICU Intensive care unit, RRT Renal replacement therapy

Data are presented as median and interquartile range for continuous variables and exact number (\%) for categorical variables. $P$-values were calculated using

Pearson's chi-square test or the Wilcoxon test. For APACHE II score, $n=93$ with MOD and $n=37$ without MOD

However, it is cumbersome to accurately predict organ failure and mortality using a single biomarker alone; therefore, a combination of biomarkers and severity scores has been proposed to provide better results. Oberholzer et al. suggested that IL-6 and APACHE II scores were correlated and showed that these combined models increased the accuracy of predicting mortality in patients with severe sepsis [34]. In addition, a study examining factors that predicted mortality 90 days after ICU admission found that combining SAPS II with IL-6 and soluble suppression of tumorigenesis-2 improved prediction accuracy [35].

The accuracy of predicting post-hospital organ failure and in-hospital mortality using qSOFA has been previously reported [36, 37]. In contrast, a previous study reported that positive qSOFA scores had high specificity but poor sensitivity for predicting inhospital mortality, acute organ dysfunction, and ICU admission in patients with infection outside the ICU, and the limits of qSOFA accuracy of the predictions have been reported [38]. The model individually added 
Table 2 Blood levels of each biomarker in patients with or without multiple organ dysfunction on day 0,1,2

\begin{tabular}{|c|c|c|c|}
\hline Day-0 & All $(n=161)$ & MOD $(n=99)$ & without MOD $(n=62)$ \\
\hline Interleukin-6, pg/ml, median (IQR) & $371.7(151.7-2471)$ & $1111(175.7-6204)$ & $210.7(87.34-554.18)$ \\
\hline Procalcitonin, ng/ml, median (IQR) & $2.19(0.34-9.3)$ & $5.18(1.27-38.08)$ & $0.43(0.15-2.12)$ \\
\hline C-reactive protein, mg/dl, median (IQR) & $10(1-21.3)$ & $12(2.47-18.55)$ & $3.95(0.28-13.78)$ \\
\hline White blood cell, $10^{3} / \mu \mathrm{L}$, median (IQR) & $13.2(6.8-18.7)$ & $11.1(4.75-18.55)$ & $15.15(11.63-19.2)$ \\
\hline Interleukin $-8, \mathrm{pg} / \mathrm{ml}$, median (IQR) & $85.3(23.88-335)$ & $193.5(54.35-965.5)$ & $36.65(15.95-93.1)$ \\
\hline Interleukin-10, pg/ml, median (IQR) & $10(3-45)$ & $17(5-75.25)$ & $5(2-16)$ \\
\hline Tumor necrosis factor- $\alpha, \mathrm{pg} / \mathrm{ml}$, median (IQR) & $3.91(1.68-8.94)$ & $5.1(2.47-12.8)$ & $2.33(1.21-4.11)$ \\
\hline Day-1 & All $(n=161)$ & $\operatorname{MOD}(n=102)$ & without MOD $(n=59)$ \\
\hline Interleukin-6, pg/ml, median (IQR) & $150.7(57.99-1096)$ & $394.6(92.83-2163.25)$ & $62.8(38.4-137.85)$ \\
\hline Procalcitonin, ng/ml, median (IQR) & $3.3(0.96-19.75)$ & $7.19(1.83-34.82)$ & $1.61(0.49-5)$ \\
\hline C-reactive protein, mg/dl, median (IQR) & $12(6.79-21.3)$ & $14.75(7.72-25.53)$ & $9.9(5.85-17.05)$ \\
\hline White blood cell, $10^{3} / \mu \mathrm{L}$, median (IQR) & $11.9(8.3-16)$ & $11.8(7.33-15.98)$ & $11.9(9.15-16.35)$ \\
\hline Interleukin -8, pg/ml, median (IQR) & $41.1(13.2-132)$ & $89.55(24.8-299.8)$ & $14.2(9.4-44)$ \\
\hline Interleukin-10, pg/ml, median (IQR) & $4(2-12.5)$ & $7(3-24)$ & $2(2-4)$ \\
\hline Tumor necrosis factor-a, pg/ml, median (IQR) & $3.34(1.94-6.04)$ & $4.04(2.21-8.2)$ & $2.67(1.6-4.11)$ \\
\hline Day-2 & All $(n=161)$ & MOD $(n=96)$ & without MOD $(n=65)$ \\
\hline Interleukin-6, pg/ml, median (IQR) & $102.1(42.37-262.5)$ & $159.6(88.86-492.98)$ & $47.53(24.75-88)$ \\
\hline Procalcitonin, ng/ml, median (IQR) & $2.62(0.83-14.62)$ & $6.53(1.54-25.88)$ & $0.95(0.39-3.93)$ \\
\hline C-reactive protein, mg/dl, median (IQR) & $15.5(9-21.5)$ & $18.1(10.83-24.08)$ & $11.9(7-17)$ \\
\hline White blood cell, $10^{3} / \mu \mathrm{L}$, median (IQR) & $10.7(8.2-14.2)$ & $11.5(7.38-14.75)$ & $9.7(8.3-13)$ \\
\hline Interleukin -8, pg/ml, median (IQR) & $25.3(11.8-82.3)$ & $55.75(20.58-120.5)$ & $11.8(5.6-24.4)$ \\
\hline Interleukin-10, pg/ml, median (IQR) & $2(2-7)$ & $4(2-9.5)$ & $2(2-2)$ \\
\hline Tumor necrosis factor- $a, \mathrm{pg} / \mathrm{ml}$, median (IQR) & $2.93(2.02-4.75)$ & $3.7(2.35-5.42)$ & $2.27(1.77-3.17)$ \\
\hline
\end{tabular}

Table 3 Receiver operating characteristic curve analysis for prediction of multiple organ dysfunction on day 2 for various biomarkers on day 0,1

\begin{tabular}{lll}
\hline & AUC & 95\%Cl \\
\hline Day-0 & & \\
Interleukin-6 & 0.647 & $0.558-0.727$ \\
Procalcitonin & 0.728 & $0.644-0.800$ \\
C-reactive protein & 0.603 & $0.513-0.686$ \\
White blood cell & 0.563 & $0.648-0.804$ \\
Interleukin -8 & 0.717 & $0.631-0.790$ \\
Interleukin-10 & 0.619 & $0.527-0.704$ \\
Tumor necrosis factor-a & 0.633 & $0.540-0.717$ \\
Day-1 & & \\
Interleukin-6 & 0.790 & $0.711-0.852$ \\
Procalcitonin & 0.705 & $0.618-0.780$ \\
C-reactive protein & 0.610 & $0.519-0.693$ \\
White blood cell & 0.457 & $0.687-0.835$ \\
Interleukin -8 & 0.789 & $0.711-0.850$ \\
Interleukin-10 & 0.751 & $0.669-0.817$ \\
Tumor necrosis factor-a & 0.635 & $0.544-0.716$ \\
\hline AUC Area under the curve $c$ Confidence
\end{tabular}

AUC Area under the curve, $\mathrm{Cl}$ Confidence interval
IL-6, IL-8, and PCT to qSOFA improved the accuracy of predicting early MOD in this study. Although CRP is a biomarker that is routinely used in clinical practice, it was excluded from the combination model with qSOFA because of its low AUC. Since qSOFA does not require testing, the improvement in predictive accuracy obtained without increasing healthcare costs is a major advantage. The combination in this study was qSOFA on admission and IL-6 on Day 1, which is also in line with that used in clinical practice. If qSOFA is positive on admission, it can be worthwhile to perform additional tests for IL-6,8 and PCT to predict early MOD. We also modeled qSOFA on Day 1 because even if qSOFA is negative on admission, it may be screened again on the next day. However, these results may be due to the fact that it is closer to Day2 when the MOD outcome is measured.

In the future, a new scoring system that combines severity scores and biomarkers to predict MODs needs to be investigated.

\section{Limitations}

This study had some limitations. First, the sample size was small partly due to the difficulty in 


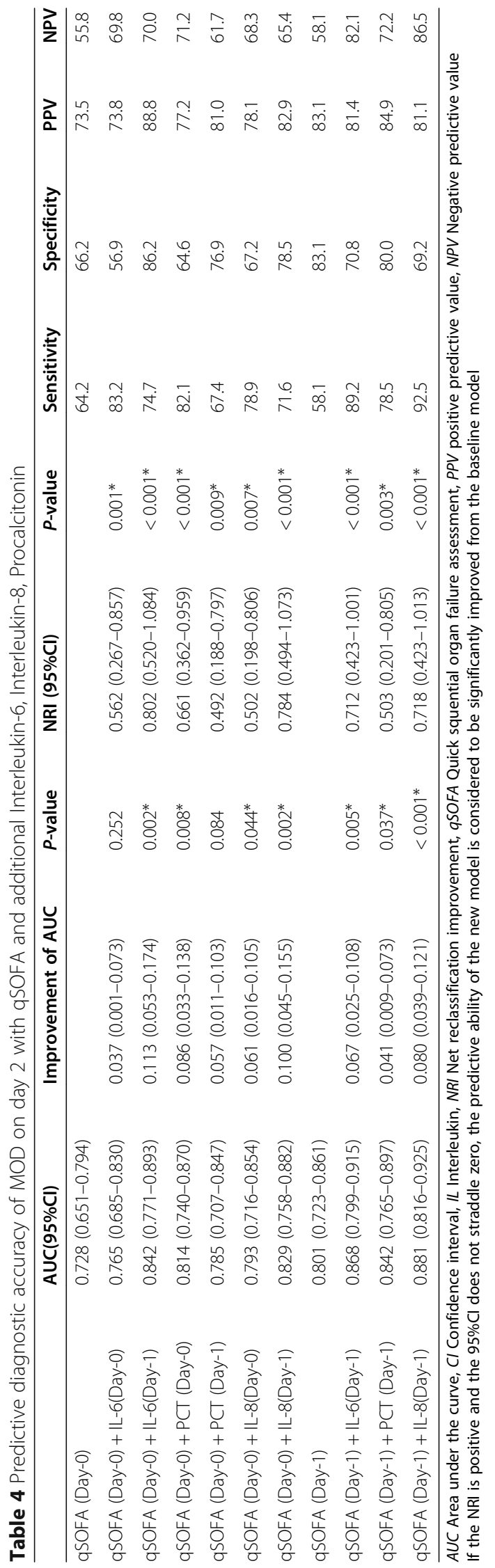



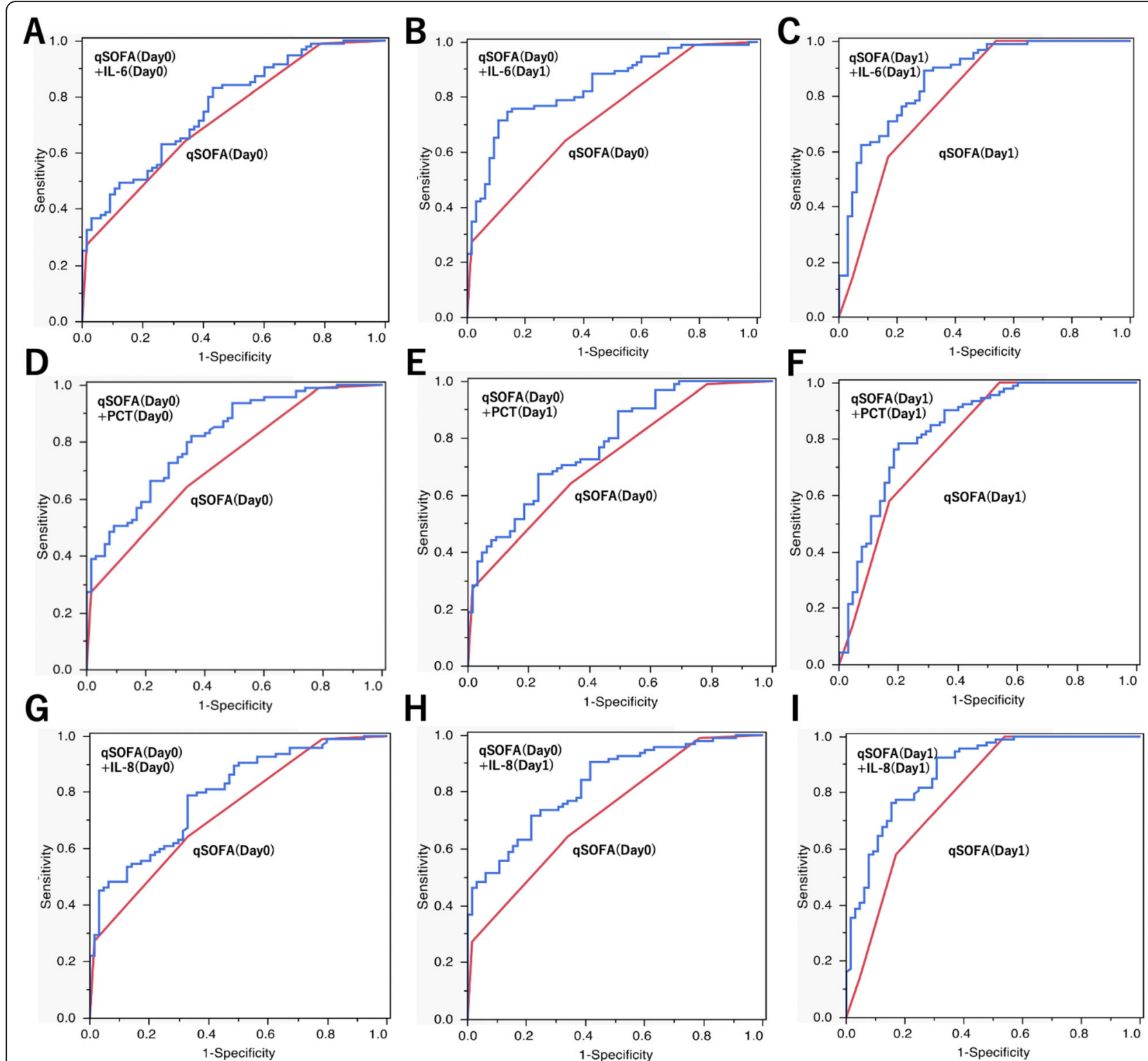

Fig. 2 Prognostic value of a combined approach of IL-6, IL-8, PCT and baseline qSOFA. A AUC for baseline qSOFA (Day 0) with IL-6 (Day 0). B AUC for baseline qSOFA (Day 0) with IL-6 (Day 1). C AUC for baseline qSOFA (Day 1) with IL-6 (Day 1). D AUC for baseline qSOFA (Day 0) with PCT (Day 0). E AUC for baseline qSOFA (Day 0) with PCT (Day 1). F AUC for baseline qSOFA (Day 1) with PCT (Day 1). G AUC for baseline qSOFA (Day 0) with IL-8 (Day 0). H AUC for baseline qSOFA (Day 0) with IL-8 (Day 1). I AUC for baseline GSOFA (Day 1) with IL-8 (Day 1). Comparison of AUC revealed that the combination model using additional serum IL-6 concentration on Days 0 and 1 had a significantly higher AUC than the baseline model that uses only the qSOFA score. AUC = area under the curve, qSOFA = quick sequential organ failure assessment, $\mathrm{IL}=$ interleukin, $\mathrm{PCT}=$ procalcitonin

obtaining consent. This study included patients with a variety of diseases. Second, one of the problems with qSOFA is that it is difficult to assess consciousness in patients with cognitive impairment before the onset of infection [39]. In addition, the blood samples after Day 1 were collected during morning period. Therefore, the timing of blood collection on Day 0 could lead to some differences.

\section{Conclusion}

We compared each biomarker as a predictor of MOD on Day 2. IL-6 on Day 1 had the highest predictive value. Furthermore, the adding IL-6, IL-8 and PCT, which had high AUC, to qSOFA score predicted MOD on Day 2 with greater accuracy. The present study showed that adding IL-6, IL-8 and PCT to qSOFA could predict early MOD. 


\section{Abbreviations}

AUC: Area under the curve; qSOFA: Quick sequential organ failure assessment; IL: Interleukin; CRP: C-reactive protein; PCT: Procalcitonin; TNF: Tumor necrosis factor; sST2: Solublesuppressionoftumorigenesis-2; MOD: Multiple organ dysfunction; NRI: Net reclassification improvement; SIRS: Systemic inflammatory response syndrome; APACHE II: Acute Physiology and Chronic Health Evaluation II; ICU: Intensive care unit

\section{Supplementary Information}

The online version contains supplementary material available at https://doi. org/10.1186/s12873-021-00534-z.

Additional file 1. Receiver operating characteristic curve analysis for prediction of multiple organ dysfunction on day 2 for various biomarkers on day 0,1 in the ICU admission group.

Additional file 2. Receiver operating characteristic curve analysis for prediction of multiple organ dysfunction on day 2 for various biomarkers on day 0,1 in the group with infection.

Additional file 3. Receiver operating characteristic curve analysis for prediction of multiple organ dysfunction on day 2 for various biomarkers on day 0,1 in the group without infection.

\section{Acknowledgements}

We thank Keisuke Yamaka, Choji Watanabe, Michiko Horie, Ai Takiyama, and Takashi Kikuchi (Roche Diagnostics K.K.) for the management of the prospective data collection.

\section{Authors' contributions}

S.I., Y.T., H.O., and M.T., conception and design, acquisition of data, analysis and interpretation of data, drafting the manuscript, and revising the manuscript critically for important intellectual content. All other authors, acquisition of data, interpretation of data, and revising the manuscript critically for important intellectual content. The author(s) read and approved the final manuscript.

\section{Funding}

This work was supported by Roche Diagnostics K.K. The funding source had no role in the study design, or preparation of the manuscript

\section{Availability of data and materials}

The data of this study are available from the authors, but restrictions apply to the availability of these data, which are not publicly available. However, data are available from the authors upon reasonable request.

\section{Declarations}

\section{Ethics approval and consent to participate}

The study was approved by the Clinical Research Ethics Committee of University of Occupational Environmental Health (Application number is H28-120), and all subjects provided informed consent, and written informed consent was obtained from all subjects prior to participation. Furthermore, all methods were performed in accordance with the relevant guidelines and regulations.

\section{Consent for publication}

This manuscript, including tables and figures, has not been published elsewhere and is not under consideration by another journal. All authors have approved the manuscript and agree with submission.

\section{Competing interests}

Authors T.S., T.N., H.O., M.N., T.M., and T.S. received honoraria for advisory board from Roche Diagnostics K.K., and authors T.M., O.T., K.M., and S. O received honoraria for advisory board and research funding for this study from Roche Diagnostics K.K. Authors R.Y., M.Y., and Y.T. declare no conflict of interests for this article.

\section{Author details}

'Department of Emergency Medicine, University of Occupational and Environmental Health, 1-1 Iseigaoka, Yahata-nishi, Kitakyushu 807-8555,
Japan. ${ }^{2}$ Department of Emergency and Critical Care Medicine, Chiba University Graduate School of Medicine, Chiba, Japan. ${ }^{3}$ Department of Emergency and Critical Care Medicine, Kurume University School of Medicine, Kurume, Japan. ${ }^{4}$ Department of Emergency and Critical Care Medicine, University of Yamanashi, Faculty of Medicine, Yamanashi, Japan. ${ }^{5}$ Department of Emergency and Critical Care Medicine, Keio University School of Medicine, Tokyo, Japan.

Received: 21 April 2021 Accepted: 29 October 2021

Published online: 08 November 2021

\section{References}

1. Jaffer U, Wade RG, Gourlay T. Cytokines in the systemic inflammatory response syndrome: a review. HSR Proc Intensive Care Cardiovasc Anesth. 2010:2(3):161-75.

2. Umegaki $T$, Ikai $H$, Imanaka $U$. The impact of acute organ dysfunction on patients' mortality with severe sepsis. Anaesthesiol Clin. 2011;27:180-4

3. Barie PS, Hydo LJ, Fischer E. Utility of illness severity scoring for prediction of prolonged surgical critical care. J Trauma. 1996;40(4):513-8. https://doi. org/10.1097/00005373-199604000-00002.

4. Peres Bota D, Melot C, Lopes Ferriera F, Nguyen Ba V, Vincent J-L. The multiple organ dysfunction score (MODS) versus the sequential organ failure assessment (SOFA) score in outcome prediction. Intensive Care Med. 2002;28(11):1619-24. https://doi.org/10.1007/s00134-002-1491-3.

5. Vincent J-L, Moreno R, Takala J, Willatts S, Mendonça A, Bruining H, et al. The SOFA (Sepsis-related organ failure assessment) score to describe organ dysfunction/failure. Intensive Care Med. 1996;22(7):707-10. https://doi.org/1 0.1007/BF01709751.

6. Singer M, Deutschman CS, Seymour CW, Shankar M, Annane D-L, Bellomo $R$, et al. The third international consensus definitions for Sepsis and septic shock (Sepsis-3). JAMA. 2016;315(8):801-10. https://doi.org/10.1001/jama.201 6.0287

7. Machado FR, Assunção MS, Cavalcanti AB, Japiassú AM, Azevedo LC, Iliveira $M C$, et al. Getting a consensus. Advantages and disadvantages of Sepsis 3 in the context of middle-income settings. Rev Bras Ter Intensiva. 2016;28(4): 361-5. https://doi.org/10.5935/0103-507X.20160068.

8. Seymour CW, Liu VX, Iwashyna TJ, Brunkhorst FM, Rea TD, Scherag A, et al. Assessment of clinical criteria for Sepsis. For the third international consensus Definitionsf or Sepsis and septic shock (Sepsis-3). JAMA. 2016; 315(8):762-74. https://doi.org/10.1001/jama.2016.0288.

9. Finkelsztein EJ, Jones DS, Ma KC, Pabón MA, Delgado T, Nakahira K, et al. Comparison of qSOFA and SIRS for predicting adverse outcomes of patients with suspicion of sepsis outside the intensive care unit. Crit Care. 2017;21(1): 73. https://doi.org/10.1186/s13054-017-1658-5.

10. Dorsett M, Kroll M, Smith CS, Asaro P, Liang SY, Moy HP. qSOFA has poor sensitivity for prehospital identification of severe sepsis and septic shock. Prehosp Emerg Care. 2017;21(4):489-97. https://doi.org/10.1080/10903127.2 016.1274348.

11. Andaluz D, Ferrer R. SIRS, qSOFA, and organ failure for assessing sepsis at the emergency department. J Thorac Dis. 2017:9(6):1459-62. https://doi. org/10.21037/jtd.2017.05.36.

12. Tanaka T, Narazaki M, Kishimoto T. IL-6 in inflammation, immunity, and disease. Cold Spring Harb Perspect Biol. 2014;6(10):a016295. https://doi.org/1 $0.1101 /$ cshperspect.a016295.

13. Oda S, Hirasawa H, Shiga H, Nakanishi K, Matsuda K, Nakamura M. Sequential measurement of IL-6 blood levels in patients with systemic inflammatory response syndrome (SIRS)/sepsis. Cytokine. 2005;29(4):169-75. https://doi.org/10.1016/j.cyto.2004.10.010.

14. Abe R, Oda S, Sadahiro T, Nakamura M, Hirayama Y, Tateishi Y, et al. Gramnegative bacteremia induces greater magnitude of inflammatory response than gram-positive bacteremia. Crit Care. 2010;14(2):R27. https://doi.org/1 $0.1186 /$ cc8898.

15. Herzum I, Renz H. Inflammatory markers in SIRS, sepsis and septic shock Curr Med Chem. 2008;15(6):581-7. https://doi.org/10.2174/092986708783 769704

16. Shimazui T, Nakada T, Yazaki M, Mayumi T, Takasu O, Matsuda K, et al. Blood interleukin-6 levels predict multiple organ dysfunction in critically ill patients. Shock. 2021;55(6):790-5. https://doi.org/10.1097/SHK 0000000000001678

17. Bone RC, Balk RA, Cerra FB, Dellinger RP, Fein AM, Knaus WA et al. Definitions for sepsis and organ failure and guidelines for the use of 
innovative therapies in sepsis. The ACCP/SCCM consensus conference committee. American College of Chest Physicians/Society of Critical Care Medicine. Chest. 1992;101(6):1644-55. https://doi.org/10.1378/ chest.101.6.1644.

18. Bozza FA, Salluh Jl, Japiassu AM, Soares M, Assis EF, Gomes RN, et al. Cytokine profiles as markers of disease severity in sepsis: a multiplex analysis. Crit Care. 2007;11(2):R49. https://doi.org/10.1186/cc5783.

19. Bouadma L, Luyt CE, Tubach F, Alvarez A, Schwebel C, Schortgen F, et al. Use of procalcitonin to reduce patients' exposure to antibiotics in intensive care units (PRORATA trial): a multicentre randomised controlled trial. Lancet. 2010;375(9713):463-74. https://doi.org/10.1016/S0140-6736(09)61879-1.

20. Tang $\mathrm{H}$, Huang $\mathrm{T}$, Jing J, Shen $\mathrm{H}$, Cui W. Effect of procalcitoninguided treatment in patients with infections: a systematic review and meta-analysis. Infection. 2009;37(6):497-507. https://doi.org/10.1007/s15010-009-9034-2.

21. Meisner M. Procalcitonin : Erfahrungen mit einer neuen Messgroesse fuer bakterielle Infek- tionen und systemische Inflammation. J Lab Med. 1999;23: 263-72.

22. Brauner JS, Rohde $L E$, Clausell N. Circulating endothelin-1 and tumor necrosis factor-a: early predictors of mortality in patients with septic shock. Intensive Care Med. 2000;26(3):305-13. https://doi.org/10.1007/ s001340051154

23. DeLong ER, DeLong DM, Clarke-Person DL. Comparing the areas under two or more correlated receiver operating characteristic curves: A nonparametric approach. Biometrics. 1988;44(3):837-45. https://doi.org/10.2307/2531595.

24. Pencina MJ, D'Agostino RB, D'Agostino RB, Vasan RS. Evaluating the added predictive ability of a new marker: from area under the ROC curve to reclassification and beyond. Stat Med. 2008;27(2):157-72. https://doi.org/10.1 002/sim.2929.

25. Jawa RS, Anillo S, Huntoon K, Baumann H, Kulaylat M. Interleukin-6 in surgery, trauma, and critical care part II: clinical implications. J Intensive Care Med. 2011;26(2):73-87. https://doi.org/10.1177/0885066610384188.

26. Pierrakos C, Vincent JL. Sepsis biomarkers: a review. Crit Care. 2010;14(1):R15. https://doi.org/10.1186/cc8872.

27. Uzzan B, Cohen R, Nicolas P, Cucherat M, Perret GY. Procalcitonin as a diagnostic test for sepsis in critically ill adults and after surgery or trauma: a systematic review and meta-analysis. Crit Care Med. 2006;34(7):1996-2003. https://doi.org/10.1097/01.CCM.0000226413.54364.36.

28. Fraunberger $P$, Wang $Y$, Holler E, Parhofer KG, Nagel D, Walli AK, et al. Prognostic value of interleukin 6 , procalcitonin, and C-reactive protein levels in intensive care unit patients during first increase of fever. Shock. 2006; 26(1):10-2. https://doi.org/10.1097/01.shk.0000215319.06866.bd.

29. Lin S, Huang Z, Wang M, Weng Z, Zeng D, Zhang Y, et al. Interleukin-6 as an early diagnostic marker for bacterial sepsis in patients with liver cirrhosis. J Crit Care. 2015;30(4):732-8. https://doi.org/10.1016/j.jcrc.2015.03.031.

30. Mokart D, Merlin M, Sannini A, Brun JP, Delpero JR, Houvenaeghel G, et al. Procalcitonin, interleukin 6 and systemic inflammatory response syndrome (SIRS): early markers of postoperative sepsis after major surgery. $\mathrm{Br} J$ Anaesth. 2005;94(6):767-73. https://doi.org/10.1093/bja/aei143.

31. Harbarth S, Holeckova K, Froidevaux C, Pittet D, Ricou B, Grau GE, et al. Diagnostic value of procalcitonin, interleukin-6, and interleukin-8 in critically ill patients admitted with suspected sepsis. Am J Respir Crit Care Med. 2001; 164(3):396-402. https://doi.org/10.1164/ajrccm.164.3.2009052.

32. Jekarl DW, Lee SY, Lee J, Park YJ, Kim Y, Park JH, et al. Procalcitonin as a diagnostic marker and IL-6 as a prognostic marker for sepsis. Diagn Microbiol Infect Dis. 2013;75(4):342-7. https://doi.org/10.1016/.dia gmicrobio.2012.12.011.

33. Mat-Nor MB, MD Ralib A, Abdulah NZ, Pickering JW. The diagnostic ability of procalcitonin and interleukin-6 to differentiate infectious from noninfectious systemic inflammatory response syndrome and to predict mortality. J Crit Care. 2016;33:245-51. https://doi.org/10.1016/j.jcrc.2016. 01.002 .

34. Oberholzer A, Souza SM, Tschoeke SK, Oberholzer C, Abouhamze A, Pribble JP, et al. Plasma cytokine measurements augment prognostic scores as indicators of outcome in patients with severe sepsis. Shock. 2005;23(6):488-93.

35. Dieplinger B, Egger M, Leitner I, Firlinger F, Poelz W, Lenz K, et al. Interleukin 6, galectin 3, growth differentiation factor 15, and soluble ST2 for mortality prediction in critically ill patients. J Crit Care. 2016;34:38-45. https://doi.org/1 0.1016/j.jcrc.2016.03.020.

36. Williams JM, Greenslade JH, McKenzie JV, Chu K, Brown AF, Lipman J. SIRS, qSOFA and organ dysfunction: insights from a prospective database of emergency department patients with infection. Chest. 2017;151(3):586-96. https://doi.org/10.1016/j.chest.2016.10.057.

37. Freund Y, Lemachatti N, Krastinova E, Laer MV, Claessens Y-E, Avondo A, et al. Prognostic accuracy of sepsis-3 criteria for in-hospital mortality among patients with suspected infection presenting to the emergency department. JAMA. 2017;317(3):301-8. https://doi.org/10.1001/jama.2016.20329.

38. Song JU, Sin CK, Park HK, Shim SR, Lee J. Performance of the quick sequential (sepsis-related) organ failure assessment score as a prognostic tool in infected patients outside the intensive care unit: A systematic review and meta-analysis. Crit Care. 2018;22(1):28. https://doi.org/10.1186/s13054-01 8-1952-x.

39. Haydar S, Spanier M, Weems P, Wood S, Strout T. Comparison of QSOFA score and SIRS criteria as screening mechanisms for emergency department sepsis. Am J Emerg Med. 2017;35(11):1730-3. https://doi.org/10.1016/j.jem.2 017.07.001.

\section{Publisher's Note}

Springer Nature remains neutral with regard to jurisdictional claims in published maps and institutional affiliations.
Ready to submit your research? Choose BMC and benefit from:

- fast, convenient online submission

- thorough peer review by experienced researchers in your field

- rapid publication on acceptance

- support for research data, including large and complex data types

- gold Open Access which fosters wider collaboration and increased citations

- maximum visibility for your research: over $100 \mathrm{M}$ website views per year

At $\mathrm{BMC}$, research is always in progress.

Learn more biomedcentral.com/submissions 